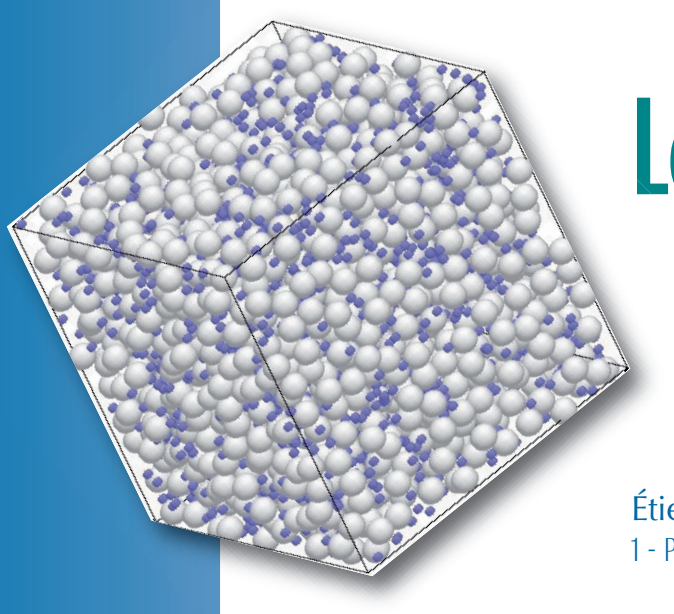

Les méthodes de la physique statistique ont rencontré la physique du solide dans ce que l'on a qualifié de matière mal condensée. Les résultats obtenus ont trouvé un prolongement dans des situations de désordre à plus grande échelle : matériaux granulaires ou poreux, colloïdes et suspensions, gels... Les deux encarts de cet article, ainsi que les deux articles suivants de ce numéro, en donnent des exemples.

Une communauté ouverte s'est constituée autour des MIAMs (milieux aléatoires macroscopiques) il y a une trentaine d’années et reste bien vivante et renouvelée dans la diversité des thèmes traités. Son histoire montre l'impact de structures légères et ouvertes.

\section{Les MIAMs,}

oul'histoire d'une communauté scientifique ouverte

Étienne Guyon1 (guyon@pmmh.espci.fr) et Daniel Bideau²

1 - Professeur émérite, PMMH, ESPCI. 2 - Professeur émérite, Université de Rennes 1. un accroissement considérable. Dans les années 1970, plusieurs rapports de prospective accompagnent ses développements, en recommandant aussi bien des actions tournées vers les applications des matériaux (J. P. Causse) que des études fondamentales (J. Friedel). L'utilisation de l'ultravide et de spectroscopies fines permet alors de mieux connaître les solides " propres " (monocristaux, surfaces propres à l'échelle atomique). Dans le même temps, le développement d'études sur les amorphes et les verres, la découverte de la localisation quantique, l'utilisation de la percolation et des fractals ouvrent la physique vers les milieux désordonnés. De Gennes, qui fut un des inventeurs de la percolation, écrit alors : «Le désordre à l'échelle microscopique est aujourd'hui un animal bien
La physique des solides a connu après-guerre apprivoisé ; ce n'est pas le cas du désordre à l'échelle macroscopique. " Des Écoles des Houches sur la matière mal condensée (1978) et sur la physique des défauts (1980) contribuent à ouvrir cette porte.

C'est avec la complicité de Pierre-Gilles et profitant d'une réorganisation engagée à la suite d'une crise universitaire à Marseille (1973) que, avec notre laboratoire de l'ESPCI puis avec le Groupe Matière Condensée et Matériaux de l'Université de Rennes, nous avons mis en place cette communauté des MIlieux Aléatoires Macroscopiques (ou, plus brièvement, MIAM), très active depuis près de trente ans. Nous avons profité, récemment, de la fin du GDR MIDI (milieux dispersés) en 2008 - héritier direct des premières structures - pour faire un point détaillé sur ces trente ans d'histoire
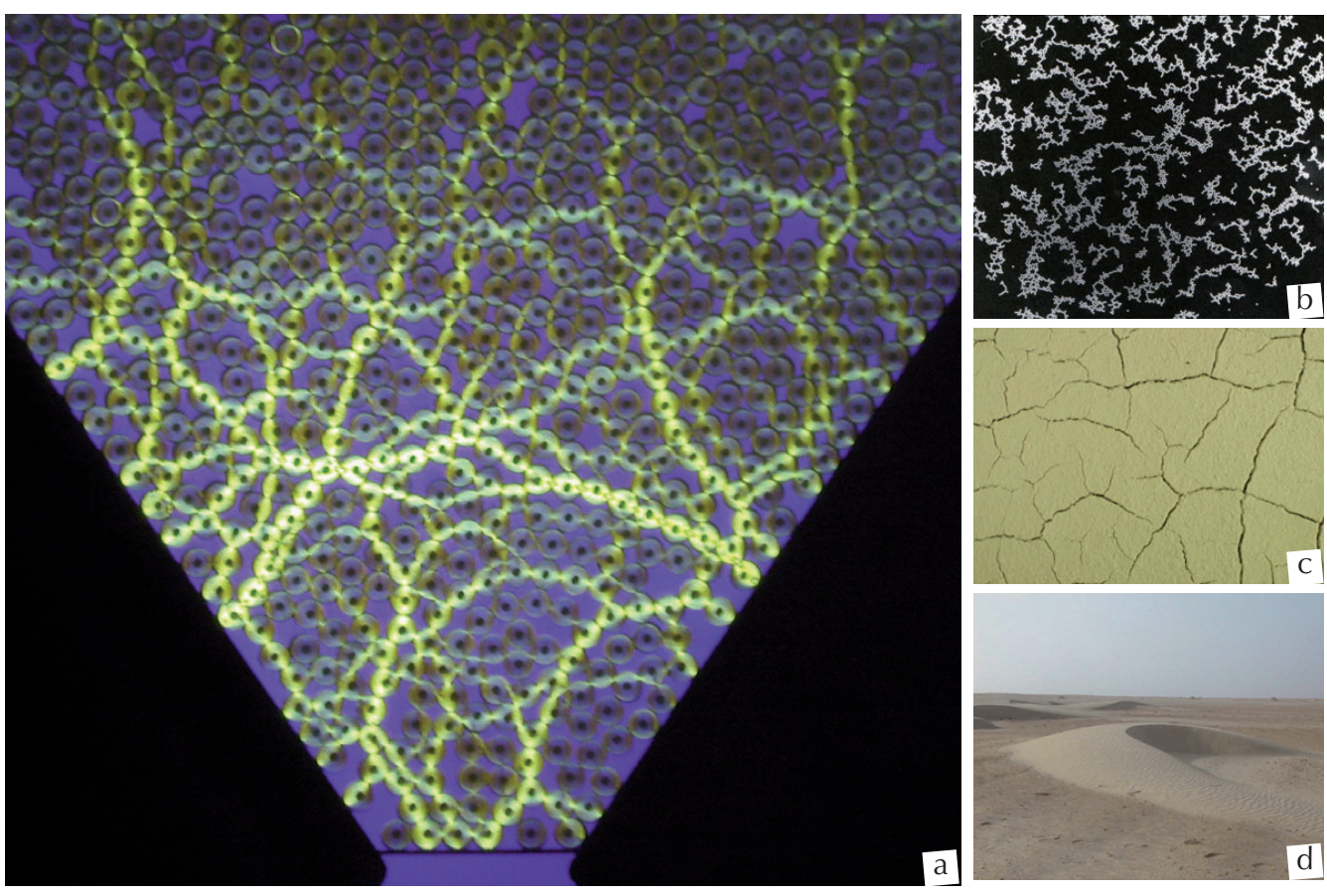

1. Quelques exemples de milieux aléatoires macroscopiques. (a) Visualisation par élasticimétrie des chaînes de forces dans un empilement de cylindres transparents sous contrainte. (Document F. Melo.) (b) Agrégat formé par des particules de cire flottant sur une surface liquide. (Document M. Cloitre, C. Allain.) (c) Fissures produites par le séchage d'une couche d’argile. (Document K.J. Maloy.) (d) Dune en croissant (barkhane). (Document F. Rioual.) (Voir aussi en couverture l'image d'une coulée de sable en avalanche.) En haut de la page : Simulation numérique de la structure d'un empilement de sphères de deux diamètres différents. (Document P. Richard.) Deux autres exemples sont les particules en suspension dans un liquide (article pp. 8-11) et les polymères renforcés par des particules (article pp. 12-15). 


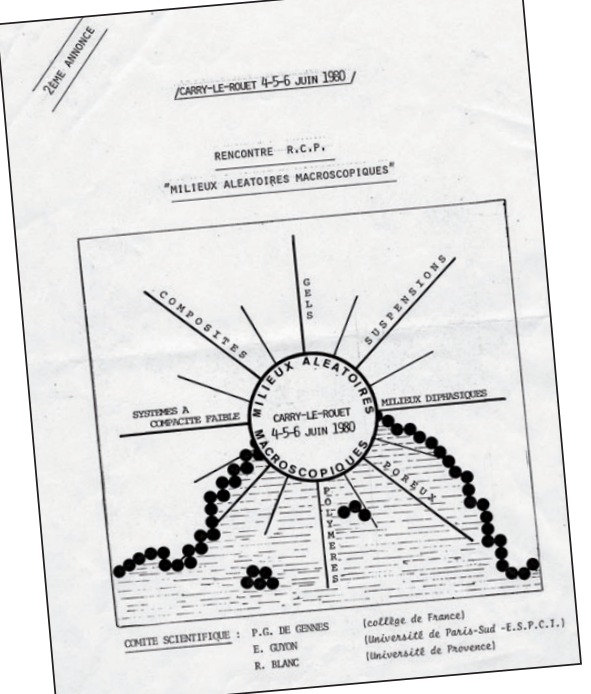

4. Annonce de la rencontre de la RCP MIAM de 1980 à Carry-le-Rouet.

modestes accordés par le CNRS et le ministère de la Recherche. Sans être en compétition avec des Écoles du type les Houches et Cargèse, auxquelles ont d'ailleurs contribué nombre des membres de cette communauté, le style beaucoup plus souple et la continuité pluriannuelle de l'esprit des rencontres, par-delà le renouvellement des thèmes année après année, a permis un très grand brassage et la constitution facile de collaborations sous forme de projets d'études, de visites, de contrats, d'obtention d'emplois et de stages. Ceci n'a pas empêché l'organisation de nombreuses écoles thématiques ouvertes à une communauté plus large [2].

L'action des rencontres MIAM a été accompagnée successivement par des actions thématiques programmées du PIRMAT dirigé par J. Hanus entre 1980 et 1990, et par les GDRs "Physique des Milieux Hétérogènes Complexes » jusqu'en 2000 et "Milieux dispersés »(MIDI) ensuite. Ces actions, sous des directions diverses, ont également largement contribué à soutenir cette communauté, aussi hétérogène que les milieux qu'elle étudie !

La gazette "info miam ", diffusée sur support papier et, elle aussi, produite de façon artisanale et à la demande, a paru continûment depuis 1980. Elle a été remplacée vers 2000 par une gazette électronique (toujours appelée "info miam »), qui est diffusée aujourd'hui à plus de 200 inscrits.

Le site du GDR MIDI est toujours très fréquenté (www.lmgc.univ-montp2.fr/MIDI).

On pourrait poser la question des applications pratiques tangibles de ces années d'activité. L'étude des nouveaux bétons, par exemple, a utilisé les résultats sur les problèmes d'empilements de grains (encadré 1, p. 6) ; celle de la dynamique des dunes, des études d'avalanches (image de couverture) et de transport éolien de grains. Les milieux pétroliers et d'hydrogéologie ont bénéficié de la meilleure connaissance des milieux poreux et fissurés (encadré 2 ci-dessous). Les études de surfaces et d'interfaces rugueuses ont permis des progrès sur la compréhension du mouillage et de la friction... Un point d'orgue a été l'article collectif du GDR MIDI sur la rhéologie des écoulements granulaires denses [3], qui est bien cité au niveau international.

Mais il faut aussi souligner l'action pédagogique constante à travers des écoles de formation, des livres, des expositions itinérantes qui ont toutes connu un grand succès...

Les MIAMs viennent d'être renouvelés par le lancement de nouveaux programmes [4]. Sans doute, les thèmes ont fortement évolué, tout en restant centrés sur la matière en désordre ; les acteurs principaux sont aussi, pour une bonne part, nouveaux. Mais une même vision de cette physique légère, soucieuse d'applications "à la de Gennes ", est toujours présente.

Dans les deux articles suivants, on décrit plus en détail deux cas importants et très différents de milieux aléatoires macroscopiques : la sédimentation d'un nuage de particules dans un liquide ("Des particules qui se la coulent douce... », pp. 8-11), et la structure et les propriétés mécaniques de matériaux composites d'intérêt industriel ("Structure multiéchelles et propriétés des matériaux du pneu », pp. 12-15).

\section{MAMs, fissures et chenoux}

L'étude de matériaux complexes a grandement bénéficié des approches statistiques de la physique du désordre. Un bon exemple est celui des roches ou matériaux compacts fracturés (massifs granitiques par exemple), à travers lesquels l'eau (ou tout autre fluide) ne peut s'écouler qu'en empruntant un réseau de fissures. Les applications sont nombreuses tant en hydrologie, en géothermie ou en génie pétrolier que dans les procédés de confinement ou de stockage de déchets (chimiques ou radioactifs, par exemple).

Un des problèmes majeurs avec ces matériaux est l'effet de « chenalisation » : les écoulements ne sont pas distribués uniformément, mais concentrés dans quelques chemins préférentiels. Cette structuration apparaît aussi bien à l'échelle de la fracture individuelle qu'à celle du réseau de fractures. Nous nous sommes surtout intéressés au premier cas, où les hétérogénéités d'écoulement sont largement liées à la rugosité des deux parois de la fissure : leur surface a une géométrie souvent caractérisée par une très large distribution de la taille des rugosités (l'encart de la figure en montre un exemple). Ces rugosités ont pour conséquence des variations d'ouverture présentes, par exemple, si au départ les deux parois sont en contact et se décalent latéralement lorsque la fracture se propage : dans ce dernier cas, les expériences et modélisations montrent qu'il apparaît des chenaux perpendiculaires au déplacement relatif.

La figure ci-contre montre les conséquences de la présence d'un tel chenal sur le déplacement d'un fluide (en bleu) par un autre, miscible avec le premier (en rouge). Le déplacement est beaucoup plus rapide dans les chemins préférentiels. De tels effets de digitation peuvent réduire de manière importante l'efficacité de la récupération du pétrole (ou de la chaleur) dans l'exploitation d'un champ pétrolifère (ou la géothermie).

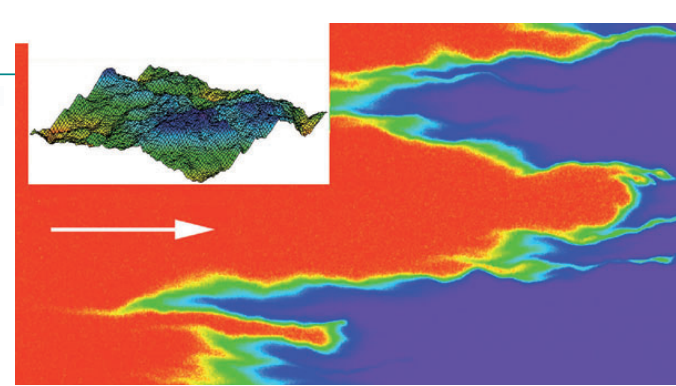

Vue en fausses couleurs des variations de concentration lors du déplacement d'un fluide (en bleu) par un autre, miscible avec le premier (en rouge), dans une fracture rugueuse " chenalisée " dans la direction parallèle à l'écoulement moyen (flèche blanche).

Les concentrations relatives des deux fluides, intermédiaires entre 0 et 1, sont marquées par les autres couleurs dans l'ordre de l'arc-en-ciel. Le champ réel de l'image est de 80 × $230 \mathrm{~mm}$. Encart : vue en perspective 3D de la surface auto-affine d'une des parois ; les hauteurs locales sont codées en fausses couleurs (bleu : hauteur élevée, rouge : faible). Pour une roche réelle la taille de l'échantillon serait de l'ordre de $100 \mathrm{~mm}$ et la hauteur des rugosités les plus grandes de l'ordre de $10 \mathrm{~mm}$.

Expériences réalisées au laboratoire FAST (UMR 7608) par A. Boschan, H. Auradou et J.P. Hulin, avec la coopération de I. Ippolito et R. Chertcoff (Université de Buenos-Aires). 